Pacific Journal of Mathematics

ENUMERATION OF THE QUASISIMPLICIAL 3-SPHERES AND
4-POLYTOPES WITH EIGHT VERTICES 


\title{
ENUMERATION OF THE QUASISIMPLICIAL 3-SPHERES AND 4-POLYTOPES WITH EIGHT VERTICES
}

\author{
Amos Altshuler ANd Leon Steinberg
}

\begin{abstract}
A complete enumeration is given for quasisimplicial 3-spheres with eight vertices. It is found that there are 661 spheres, and it is proved that precisely 20 of these are not polytopal.
\end{abstract}

1. Introduction. This work is the first part of an enumeration of all the combinatorial 3-spheres and the 4-polytopes with 8 vertices. It is devoted to the quasisimplicial cases; that is, to those 3-spheres and 4-polytopes all of whose facets are simplicial.

In the last two decades extensive work has been done on the enumeration of simplicial 3-spheres and simplicial 4-polytopes with 8 vertices ([15], [10], [1]), with 9 vertices $([4],[6],[7],[8])$ and with 10 vertices (the neighborly cases only, see [3]). With regard to the general (that is, not necessarily simplicial) cases, Kleinschmidt [16] proved that every $d$-sphere with up to $d+4$ vertices is polytopal, Reif ([19]) prepared a detailed list of the 31 4-polytopes with 7 vertices and a partial list (containing some 1050 cases) of 3-spheres with 8 vertices, and Perles ([14, pages 114, 424]) determined the number of $d$-polytopes with $d+3$ vertices for $d \leq 6$.

The simplicial 3-spheres with 8 vertices are well known ([15], [10], [1]). There are 39 , exactly two of which are non-polytopal. The pyramidal 3 -spheres with 8 vertices are also well known, based on the determination by Hermes, quoted in [13] and [14, page 424], of the 3-polytopes with 7 vertices. Obviously they all are polytopal, and their number is 34 . Five of them are quasisimplicial.

In the present work we find all the non-simplicial, non-pyramidal quasisimplicial 3-spheres with 8 vertices. It turns out that there are 617, exactly 18 of which are not polytopal. Therefore the total number of quasisimplicial 3-spheres with 8 vertices is 661 of which 20 are not polytopal. Our 18 non-polytopal spheres are described in detail in Table 1. A detailed description of all the 617 cases is of course beyond the scope of a single paper. The full catalogue of these cases can be obtained upon request from the second author. 
The non-polytopal spheres are of special interest because they might help to solve the classical "Steinitz problem", concerning the combinatorial characterization of the $d$-polytopes (for $d>3$ ) among the $(d-1)$ spheres. The first non-simplicial non-polytopal 3-sphere with 8 vertices was discovered in [11], Kleinschmidt [17] found two more such spheres. Recently Schulz ([20]) prepared a list of 27 non-polytopal 3-spheres with 8 vertices, which contains the 5 cases previously known. Of those 27 , ten are quasisimplicial but not simplicial. They are among the 18 cases listed in Table 1 .

In $\S 2$ we introduce the basic concepts involved in the present work. In $\S 3$ we describe the construction of the 617 quasisimplicial, non-simplicial, non-pyramidal 3-spheres with 8 vertices. In $\$ 4$ we describe the theoretical background needed for the classification of the 617 spheres into polytopes and non-polytopes. In $\$ 5$ we prove that the 18 spheres listed in Table 1 are not polytopal. In $\$ 6$ we quote several theorems from [5] with which we prove the polytopality of all but three of the remaining 599 spheres. In $\$ 7$ we prove that these three spheres are polytopal also and we conclude in $\S 8$ with some remarks.

Our terminology follows [14] and [7].

2. Basic concepts. A d-cell complex is a finite collection $\mathcal{C}$ of $k$-cells, $0 \leq k \leq d$, such that

1. each $d$-cell $F$ is associated with a convex $d$-polytope $P(F)$ and with a (piecewise linear) homeomorphism $h(F)$ between $F$ and $P(F)$;

2. each face of a $d$-cell in $\mathcal{C}$ is a member of $\mathcal{C}$, where a face of a $d$-cell $F$ is the inverse image of a face of $P(F)$ under $h(F)$;

3. the intersection of any two members of $\mathcal{C}$ is a (possibly empty) face of both members.

A combinatorial $d$-sphere is a $d$-cell complex whose body (i.e. the union of its members) is homeomorphic to $S^{d}$. A combinatorial d-ball is similarly defined to be a $d$-cell complex whose body is homeomorphic to a topological $d$-ball.

A word of caution seems appropriate. The term combinatorial sphere appears in the literature with more than one meaning. For example in [12] the underlying complex is assumed to be geometric - that is, all the $d$-cells are assumed to be $d$-polytopes, properly embedded in a Euclidean space. We will refer to such a sphere as geometric. On the other hand, in [8], the underlying complex is assumed to be simplicial. (See also the concluding paragraph in $[9, \S 1])$. The definition given here is adopted basically from [10]. 
Obviously, every geometric $d$-sphere is a combinatorial $d$-sphere. Other examples of combinatorial $d$-spheres are provided by $d$-diagrams, as defined in [14, page 44]. The fact, established in [21], that there are geometric spheres that are not diagrams, and vice versa, shows that the concept of a combinatorial sphere is broader than the other two concepts. All three, however, agree in the case of the boundary complex of a convex polytope.

To simplify the notation, we refer to the cells of a combinatorial sphere as if they themselves were convex. Thus, using the notation of the above definitions, if $F$ is a 3-cell such that $P(F)$ is the octahedron shown in Figure 1, we say that $F$ is an octahedron, that 45 is an edge of $F$, that the diagonal 12 lies inside $F$, and so on. The convex hull of the points $a_{1}, a_{2}, \ldots, a_{n}$ is denoted by $a_{1} \cdots a_{n}$. If not otherwise specified, all the spheres mentioned here are assumed to be combinatorial and 3-dimensional.

If $a, b$ are vertices in a sphere $S$ but the edge $a b$ is not in $S$, we say that $a b$ is a missing edge of $S$. For every two vertices $a, b \in S$, we define val $a b$, the valence of the edge (missing or not) $a b$, to be the number of 3 -cells in $S$ that contain both vertices $a$ and $b$. Thus, if $a b$ is an edge, val $a b$ is as defined in [7] and is $\geq 3$, while if $a b$ is a missing edge, then $0 \leq$ val $a b \leq 2$, and val $a b=2$ iff $a, b$ belong to some 2 -face in $S$, but $a b$ is not an edge. The last case is impossible where $S$ is quasisimplicial. Also, if $a b, b c, a c$ are edges in some 3-cell $A$ of $S$ but the triangle $a b c$ is not a face of $A$ (and therefore also not a face of any 3-cell of $S$ ), we say that $a b c$ is a missing 2-face in $A$ and in $S$.

With every 3-sphere $S$ with $n$ vertices $a_{1}, \ldots, a_{n}$, we associate an $n \times n$ matrix, the edge-valence matrix of $S$, in which the $(i, j)$ element is the valence of the edge $a_{i} a_{j}$ (missing or not). This matrix, of course, depends on the particular ordering chosen for the vertices of $S$, but its determinant is independent of that ordering, (see [3, §2] and [7, \$2]) and plays an important role in the identification of a sphere. Since our interest is in the combinatorial types of spheres, we do not distinguish between spheres that are isomorphic.

Finally, if $\mathcal{C}$ is a cell-complex and $A \in \mathcal{C}$, then $\operatorname{st}(A, \mathcal{C})$, the star of $A$ in $\mathcal{C}$, is the complex $\{C \in \mathcal{C}$ : There is a cell $B \in \mathcal{C}$ such that $A$ and $C$ are faces of $B\}$, and ast $(A, \mathcal{C})$, the antistar of $A$ in $\mathcal{C}$, is the complex of all the cells in $C$ disjoint from $A$.

3. Construction. Let $2 S_{8}^{3}$ be the family of (combinatorial types of the) 3-spheres with 8 vertices which are quasisimplicial, but not simplicial and not pyramidal. Since each facet in such a sphere $S$ has at most 6 
vertices, it is either a simplex or one of the types A, B, C, shown in Figure 1. (We refer to a cell of type $\mathrm{C}$ as an octahedron, though its vertices 1,2 , 3,4 , or $3,4,5,6$, or $1,2,5,6$, do not necessarily lie in the same plane.)

A cell of type A (double tetrahedron) can be uniquely subdivided into two tetrahedra, without adding any extra vertices or edges. A cell of type $B$ can be uniquely subdivided into three tetrahedra, without extra vertices or edges. A cell of type $\mathrm{C}$ (octahedron), after adding to it one of its three main diagonals, can be uniquely subdivided into four tetrahedra, without extra vertices or edges.

By thus subdividing each facet of $S \in 2 S_{8}^{3}$ which is not a simplex, a simplicial 3-sphere $S^{\prime}$ with 8 vertices is obtained. There are 39 simplicial 3-spheres with eight vertices (see [15], [10]) which we denote by $M_{i}^{8}$, $1 \leq i \leq 39$, where for $1 \leq i \leq 37, M_{i}^{8}$ is the polytopal sphere $P_{i}^{8}$ of [15], $M_{38}^{8}$ is Grünbaum's non-polytopal sphere denoted $\Re$ in [15], and $M_{39}^{8}$ is Barnette's non-polytopal sphere ([10]). Thus $S^{\prime}$ is one of these $M_{i}^{8}$ 's. Note that if $S$ contains no octahedron, then $S^{\prime}$ is uniquely determined by $S$, while if $S$ contains an octahedron, $S^{\prime}$ may also depend on the particular diagonal chosen for the subdivision of the octahedron.

The idea used was to start with the 39 simplicial spheres $M_{i}^{8}$, and to construct the family $2 S_{8}^{3}$ from them by reversing the process of subdivision described above. The following lemma is useful:

LEMMA 1. No $S \in 2 \delta_{8}^{3}$ contains two facets with 6 vertices.

Proof. If $X, Y$ are facets of $S \in 2 \Im_{8}^{3}$ with 6 vertices, then $X \cap Y$ contains at least 4 vertices. But this is impossible, since $X$ and $Y$ are simplicial 3-polytopes. Thus, no $S \in 2 \delta_{8}^{3}$ contains two facets of type B, two facets of type $\mathrm{C}$, or a facet of type B and a facet of type C.

The reversal of the process of subdivision mentioned above, depends upon the following three operations, each of which applies to a 3-sphere $S$.

Operation A. Choose two tetrahedra, abcd and abce, in $S$ which share a common triangle $a b c$, such that de is a missing edge in $S$ with val $d e=0$, and "glue" them along the triangle $a b c$ to yield a 3-cell abcde of type A.

Operation B. Choose three tetrahedra abdf, abef and acef, in $S$ such that $b c, c d$, de are missing edges of valence 0 in $S$, and "glue" them along the triangles $a b f$, aef, to yield a 3-cell abcdef of type $B$.

Operation C. Choose an edge $a b$ of valence 4 in $S$ such that the four 3-cells containing it (abce, abde, abdf and $a b c f$ ) are tetrahedra and such 
that $c d$ and $e f$ are of valence 0 in $S$, and "glue" the four tetrahedra along the triangles $a b c, a b d$, abe, $a b f$-removing the edge $a b$-to yield a 3-cell abcdef of type C.

Using a CYBER-174 computer, we applied Operation A to each 3-sphere $M_{i}^{8}, 1 \leq i \leq 39$, in all possible ways. To each resulting sphere $S$ we again applied Operation $\mathrm{A}$ in all possible ways, and so on. By repeatedly applying Operation A to the spheres $M_{i}^{8}$ we obtained all the spheres in $2 S_{8}^{3}$ whose facets are just simplices and double tetrahedra (type A). We then applied Operation B, followed by repeated application of Operation A, to each $M_{i}^{8}$ to obtain all the spheres in $2 S_{8}^{3}$ which contain a facet of type B, in addition to simplices and double tetrahedra. Finally, Operation C, followed by Operations of type A, was applied to each $M_{i}^{8}$ to yield the remaining members in $2 S_{8}^{3}$.

For each resulting sphere $S$, the edge-valence matrix and its determinant have been determined. The spheres were then checked for isomorphism. Here, as in other works (see [6], [7], [8]), the edge-valence matrix and its determinant proved to be very helpful. Also, as follows from a previous remark, two spheres in $2 \Im_{8}^{3}$ that do not contain an octahedron (type C) can be isomorphic only if, beside sharing the same number of facets of each type and the same determinant, they result from the same simplicial 3-sphere $M_{i}^{8}$ (while two spheres that contain an octahedron may be isomorphic even if they result from different $M_{i}^{8}$ 's).

Altogether, the final list contains 617 non-isomorphic spheres, falling into three groups: first, 520 spheres $S_{i}(1 \leq i \leq 520)$ that do not contain a cell of type B nor of type $\mathrm{C}$, then 80 spheres $S_{i}(521 \leq i \leq 600)$ that contain a cell of type B and finally 17 spheres $S_{i}(601 \leq i \leq 617)$ that contain a cell of type $C$. The spheres within each group are ordered lexicographically by increasing number of cells, increasing number of double-tetrahedra and by increasing determinant. In the rare event of equal determinants the order is casual.

4. Background for the classification. The next step after constructing the 617 quasisimplicial spheres was to classify them into polytopes and non-polytopes.

Let $S$ be any of our 617 spheres, with the vertices labelled $1,2, \ldots, 8$. If $S$ is polytopal, let $P$ be a 4-polytope realizing $S$, and we assume the labelling of the vertices of $P$ to be the same as in $S$. Let $x \in\{1,2, \ldots, 8\}$ and let $Q=\operatorname{conv}($ vert $P \backslash\{x\}) . Q$ is a 4-polytope with seven vertices. Denote by $S^{x}$ the combinatorial 3-sphere isomorphic to bd $Q$. It is important to note that $S^{x}$ depends not only on $S$, on $x$ and on $S$ being polytopal, but may also depend on the particular polytope $P$ chosen to 
realize $S$. In the terminology of $[14, \S 5.2]$, the point $x$ lies beneath some facets of $Q$, beyond some other facets of $Q$, and-unlike the simplicial case-may lie in the affine hulls of some facets of $Q$.

The relation between the facial structure of $Q$ and that of $P$ is determined by Grunbaum's theorem [14, Theorem 5.2.1] (or rather by its corrected version in [5]). That is, if $\mathbb{Q}=\left\{A_{1}, \ldots, A_{r}\right\}, \mathscr{B}=\left\{B_{1}, \ldots, B_{s}\right\}$, $\mathcal{C}=\left\{C_{1}, \ldots, C_{t}\right\}$ is a partition of the facets of $Q$ such that the point $x$ lies beyond each $A_{i}, 1 \leq i \leq r$, beneath each $B_{j}, 1 \leq j \leq s$, and in aff $C_{k}$, $1 \leq k \leq t$, then the combinatorial structure of $P=\operatorname{conv}(Q \cup\{x\})$ depends only on the partition $\mathcal{Q}, \mathscr{B}, \mathcal{C}$ of the facets of $Q$. It does not depend on the actual point $x$ chosen, provided, of course, that the set of points in $R^{4}$ that lie beyond each $\mathbb{Q}_{i}$, beneath each $B_{j}$ and in the affine hull of each $C_{k}$, is not empty.

So far, both the definition and the existence of the sphere $S^{x}$ depends on $S$ being polytopal. But it is the polytopality of $S$ that we want to prove or disprove. Indeed, in the next section we will see that $S^{x}$ can be defined and constructed combinatorially, without any previous knowledge of the polytopality of $S$.

If for some vertex $x$ of $S$ it can be shown that no sphere $S^{x}$ does exist, then $S$ is not polytopal. If for every vertex $x$ of $S$ there exists a sphere which might serve as an $S^{x}$ then-fixing $x$ and $S^{x}$ and assuming $Q$ to be a polytopal realization of $S^{x}$-the partition $\mathscr{Q}, \mathscr{B}, \mathcal{C}$ of the facets of $Q$ required for constructing a polytope $P$ which is to realize $S$, is already determined by $S, x$ and $S^{x}$. (Since we assume the labelling of the proper faces of $Q$ to be the same as that of $S^{x}$, every partition of the facets of $Q$ carries over to $S^{x}$, and vice versa). Namely, $\mathscr{B}$ is the set of 3-cells common to $S$ and to $S^{x}$ (that is, all the 3-cells in ast $(x, S)$ ), $\mathcal{C}$ is the set of 3-cells $C$ of $S^{x}$ such that vert $C \cup\{x\}$ is the vertex set of some 3-cell in $S$, and $\mathbb{Q}$ is the set of all the remaining 3-cells in $S^{x}$. (Note that unlike $\mathcal{Q}, \mathscr{B}$ depends on $S$ and $x$ only, and not on $S^{x}$. The situation for $\mathcal{C}$ is more complicated and we deal with it in $\S 5$.)

It follows from the last discussion, that the following sequence of steps is required for deciding the polytopality of a sphere $S$ : Start with a vertex $x$ in $S$ and

1. construct a sphere $S^{x}$ such that

2. $S^{x}$ is polytopal, and

3. a polytope $Q$ can be found which realizes $S^{x}$, such that the set of points that lie beyond each $A \in \mathbb{Q}$, beneath each $B \in \mathscr{B}$ and in the affine hull of each $C \in \mathcal{C}$, is not empty. $(\mathcal{Q}, \mathfrak{B}, \mathcal{C}$ is the partition of the facets of $S^{x}$, uniquely determined by $S, x$ and $S^{x}$.) 
It also follows from Grünbaum's theorem that the possibility of carrying out these steps for one vertex $x$ of $S$ is sufficient to prove that $S$ is polytopal, while the impossibility of carrying out these three steps for one vertex $x$ of $S$ is sufficient to prove that $S$ is not polytopal.

In our case, since $S$ has 8 vertices and $S^{x}$ (if it exists) has 7 vertices, the second step does not pose any problem: Kleinschmidt ([16]) proved that very $d$-sphere with up to $d+4$ vertices is polytopal. Actually, Kleinschmidt deals with geometric spheres, but we have checked independently that there are 31 combinatorial 3-spheres with 7 vertices altogether, and that they coincide with the 31 4-polytopes with 7 vertices listed in [19]. The proof is simple, technical and tedious, and we skip it.

As for the first step, we will see that in 18 of the 617 spheres under consideration there is a vertex $x$ such that there exists no $S^{x}$, and therefore they are not polytopal. Concerning the third step, quite unexpectedly, we were able to show that for each of the remaining 599 spheres-all of which proved polytopal-there is a vertex $x$ and there is a sphere $S^{x}$ such that for any polytope $Q$ chosen to realize $S^{x}$, the condition in the third step is satisfied.

We turn now to the strategy for constructing $S^{x}$ or to prove its nonexistence.

5. Classification: $\mathbf{1 8}$ non-polytopal spheres. Let $S$ be a 3 -sphere and $x$ a vertex in $S$. Our purpose is to define the sphere $S^{x}$ in a way that is independent of the question whether or not $S$ is polytopal and to describe a method for finding it. Generally speaking, $S^{x}$ will be obtained by removing the complex $\operatorname{st}(x, S)$ from $S$ and refilling the "hole" thus created in $S$ with a suitable complex that uses only vertices of $S$ other than $x$. In the case where all the facets of $S$ which contain $x$ are simplices, this complex is actually the "refill" defined and studied in [7, page 100]. In the general case, however, there arise some complications which we are to explain now.

Assume for the moment that $S$ is polytopal, that $P$ is a 4-polytope realizing $S$ with the same labelling of the vertices, that $Q=$ conv(vert $P \backslash\{x\})$ and that $S^{x}$ is a 3-sphere isomorphic to bd $Q$, again with the same labelling of the vertices. (This convention allows us to treat a cell combinatorially - that is, as a member in $S$-or geometrically, as a cell in bd $Q$, according to need.) As before, let $\mathscr{Q}(\mathscr{B})$ denote the set of facets of $Q$-and of $S^{x}$-beyond (beneath) which $x$ lies, and let $\mathcal{C}$ be the set of facets of $Q$ in the affine hulls of which $x$ lies. clearly, $\mathscr{B}$ is the set of 3 -cells in ast $\{x, S\}$. 
Now let $C \in \mathcal{C}$. As a facet of $Q, C$ has the property that $C^{\prime}=$ conv(vert $C \cup\{x\}$ ) is a facet of $P$ (and of $S$ ), and vert $C^{\prime}=\operatorname{vert} C \cup\{x\}$. As a 3-cell in $S^{x}$, however, without reference to a particular polytope $P$ chosen to realize $S$, all w'c can say about $C$ is that vert $C \cup\{x\}$ is the vertex-set of some facet $C^{\prime}$ in $S$, and there is some realization of $C^{\prime}$ as a 3-polytope such that $C$ is isomorphic to conv(vert $\left.C^{\prime} \backslash\{x\}\right)$. Generally speaking, this does not define $C$ uniquely, and every such $C$ will be called an admissible 3-cell arising from $C^{\prime}$ at $x$.

As an example, let $C^{\prime}$ be the 3-cell of type $B$ in Figure 1, with $x=1$. Depending on the position of the vertices of $C^{\prime}$, there are three admissible candidates for $C$ : the 3-pyramid 24356 with apex 6 , the double tetrahedron 23645 (with order of vertices being "canonic", as in Figure 1, type A) and the double tetrahedron 45623.

We now define the concept of a refill (compare [8, page 100]) and the sphere $S^{x}$.

DEFINITION 1. Let $S$ be a non-pyramidal 3-sphere, and let $x$ be a vertex in $S$. A 3-complex $R$ is called a refill for $S$ at $x$ if

(i) the union $|R|$ of the cells in $R$ is a 3-ball,

(ii) all the vertices in $R$ are vertices in $\operatorname{ast}(x, S)$,

(iii) $R \cap$ ast $(x, S)$ is the boundary complex of both $R$ and ast $(x, S)$,

(iv) for every 3-cell $C^{\prime}$ in $\operatorname{st}(x, S)$ which is not a 3-pyramid with apex $x$, there is in $R$ an admissible 3-cell $C$ arising from $C^{\prime}$ at $x$, and

(v) $\operatorname{ast}(x, S) \cup R$ is a 3-sphere.

REMARK. The Auxiliary Theorem in [3, page 407] can be used here, as in $[3$, pages 409,410$]$, to show that condition (v) in the last definition can be replaced by

$\left(\mathrm{v}^{\prime}\right)$ No missing edge of valence $>0$ interior to $\operatorname{ast}(x, S)$ (resp. $R$ ) is an edge or a missing edge of valence $>0$ in $R$ (resp. ast $(x, S)$ ), and no missing 2-face in $\operatorname{ast}(x, S)$ (resp. $R$ ) is a 2-face or a missing 2-face in $R$ $($ resp. $\operatorname{ast}(x, S))$.

DEFINITION 2. In the notation of Definition 1 , if $R$ is a refill for $S$ at $x$, we denote the 3-sphere ast $(x, S) \cup R$ by $S^{x}$, and call it a contraction of $S$ at $x$. We also say that $S$ is directly obtainable from $S^{x}$ at $x$ (Compare [8, page 100]) and that $S$ is contractible at $x$.

Clearly, a polytopal 3-sphere $S$ has a contraction at each of its vertices, the contraction at the vertex $x$ being the boundary complex of conv(vert $P \backslash\{x\}$ ), where $P$ is any 4-polytope realizing $S$. Thus: 
THEOREM 2. If a 3-sphere $S$ has a vertex at which it is not contractible, then $S$ is not polytopal.

How can we tell if a 3-sphere $S$ is contractible at a vertex $x$ ? In other words, how can we find a refill $R$ for $S$ at $x$ ? The first step is to find the set $\mathcal{C}=\mathcal{C}(R)$ of all the 3-cells $C$ in $R$ which are the admissible 3-cells arising from those facets $C^{\prime}$ of $S$ which contain $x$ but are not pyramidal at $x$. As already mentioned, in general, a facet $C^{\prime}$ of $S$ which contains $x$ (and is not pyramidal at $x$ ) does not define a unique admissible $C$. If, however, $C^{\prime}$ is a double tetrahedron, then $C$ is unique, and it is a tetrahedron.

In each of the first 520 spheres $S_{i}$ of our list of 617 all the facets are just tetrahedra and double tetrahedra, and therefore at each vertex $x$ of $S_{i}$, the set $\mathcal{C}$ is uniquely and easily determined by $S_{i}$ and $x$. If $S_{l}$ has a refill $R$ at $x$, let $\mathscr{Q}$ denote the set of all 3-cells in $R \backslash \mathcal{C}$, and let $\mathcal{C}^{\prime}\left(\mathcal{Q}^{\prime}\right)$ denote the complex of all the faces of the members of $\mathcal{C}$ (resp. $Q$ ).

Clearly $\operatorname{bd}\left(\operatorname{ast}\left(x, S_{i}\right) \cup \mathcal{C}^{\prime}\right)=\operatorname{bd} \mathbb{Q}^{\prime}$. Since $\operatorname{bd}\left(\operatorname{ast}\left(x, S_{i}\right) \cup \mathcal{C}^{\prime}\right)$ is simplicial, we conclude that bd $\mathbb{Q}^{\prime}$ is simplicial. Thus even if $\mathbb{Q}^{\prime}$ is not simplicial, it can be simplicially subdivided, without the addition of extra vertices. (We don't know if this assertion is true in general, but it is easy to check that it is true in our case, where $\mathbb{Q}^{\prime}$ has at most seven vertices.) We may therefore assume that $\mathbb{Q}^{\prime}$ (and therefore also the entire refill $R$ ) is simplicial. Since we know the boundary complex of $Q^{\prime}$, we may invoke the algorithm described in [1] to compute $\mathbb{Q}^{\prime}$, and to actually compute all the possible simplicial complexes $\mathcal{Q}^{\prime}$. The strong restriction that no face interior to ast $\left(x, S_{i}\right) \cup \mathcal{C}^{\prime}$ can be in $\mathbb{Q}^{\prime}$, and the additional restrictions mentioned in Condition $\left(\mathrm{v}^{\prime}\right)$ make the process of computing $\mathbb{Q}^{\prime}$ very efficient.

Thus we prepared a list of all the simplicial refills for each sphere $S_{i}$, $1 \leq i \leq 520$, at each of its vertices. For 18 of those 520 spheres there was at least one vertex at which the sphere is not contractible, and therefore those 18 spheres (listed in Table 1) are not polytopal.

As an example, consider the sphere $S=S_{137}$ of Table 1 at its vertex 4 . Here the 3-cells in ast $(4, S)$ are 13567, 25718, 2356, 2358 and 3578. (In a quasisimplicial 3-sphere, a 3-cell with five vertices is necessarily a doubletetrahedron, and its representation is canonic, as in Figure 1, type A.) $\mathcal{C}$ is composed of 1256 and 2368. The 2-faces in $\operatorname{bd}\left(\operatorname{ast}(4, S) \cup \mathcal{C}^{\prime}\right)$, and therefore also in bd $\mathbb{Q}^{\prime}$, are all triangles: 136, 137, 127, 278, 378, 126, 268 and 368 , and they define a triagulation of $T$ of a 2 -sphere. We are looking for a triangulation $\mathbb{Q}^{\prime}$ of the 3-ball with boundary $T$, which uses only vertices from the set $\{1,2,3,5,6,7,8\}$. 
Since the vertex 5 and the edge 23 are interior in ast $(4, S) \cup \mathfrak{e}^{\prime}$, they should not appear in $\mathscr{Q}^{\prime}$. Also, 18 and 67 are missing edges of valence 1 in ast $(4, S) \cup \mathcal{C}^{\prime}$, and they too should not appear in $\mathbb{Q}^{\prime}$. Now, since the triangle 136 is in $T$, there must be some tetrahedron $136 y$ in $\mathbb{Q}^{\prime}$, where $y \in\{2,5,7,8\}$, but because of the above restrictions, no such $y$ is possible. We thus conclude that $S_{137}$ is not contractible at the vertex 4 , and therefore it is not polytopal.

\section{TABLE 1}

The non-polytopal, quasisimplicial non-simplicial 3-spheres with eight vertices

\begin{tabular}{|c|c|c|c|c|c|c|}
\hline $\begin{array}{l}\text { Case } S_{i} \\
i=\end{array}$ & & & 3-cells & & & Non contractible at \\
\hline $\begin{array}{l}125 \\
\text { det. }=1200\end{array}$ & $\begin{array}{l}24718 \\
15723 \\
12645\end{array}$ & $\begin{array}{r}13467 \\
1356 \\
2468\end{array}$ & $\begin{array}{l}3468 \\
2568 \\
3568\end{array}$ & $\begin{array}{l}2578 \\
3578 \\
3478\end{array}$ & & 1,8 \\
\hline $\begin{array}{l}137 \\
\text { det. }=4087\end{array}$ & $\begin{array}{l}13567 \\
25718 \\
12645\end{array}$ & $\begin{array}{r}23468 \\
1247 \\
1346\end{array}$ & $\begin{array}{l}1347 \\
2356 \\
2358\end{array}$ & $\begin{array}{l}3578 \\
2478 \\
3478\end{array}$ & & 4,5 \\
\hline $\begin{array}{l}194 \\
\text { det. }=244\end{array}$ & $\begin{array}{l}15723 \\
12645 \\
13467\end{array}$ & $\begin{array}{l}1247 \\
1356 \\
2468\end{array}$ & $\begin{array}{l}3468 \\
2568 \\
3568\end{array}$ & $\begin{array}{l}2578 \\
3578 \\
2478\end{array}$ & 3478 & 1,8 \\
\hline $\begin{array}{l}199 \\
\text { det. }=1100\end{array}$ & $\begin{array}{l}34718 \\
12467 \\
23568\end{array}$ & $\begin{array}{l}1256 \\
1257 \\
1346\end{array}$ & $\begin{array}{l}1356 \\
1357 \\
2346\end{array}$ & $\begin{array}{l}2578 \\
3578 \\
2348\end{array}$ & 2478 & 4,5 \\
\hline $\begin{array}{l}348 \\
\text { det. }=31008\end{array}$ & $\begin{array}{l}24718 \\
12645 \\
13467\end{array}$ & $\begin{array}{l}1257 \\
1356 \\
1357\end{array}$ & $\begin{array}{l}2346 \\
2368 \\
2568\end{array}$ & $\begin{array}{l}3568 \\
2578 \\
3578\end{array}$ & $\begin{array}{l}2348 \\
3478\end{array}$ & 4,5 \\
\hline $\begin{array}{l}349 \\
\text { det. }=31775\end{array}$ & $\begin{array}{l}24718 \\
12645 \\
25867\end{array}$ & $\begin{array}{l}1257 \\
1346 \\
1356\end{array}$ & $\begin{array}{l}1357 \\
1347 \\
2346\end{array}$ & $\begin{array}{l}2368 \\
3568 \\
3578\end{array}$ & $\begin{array}{l}2348 \\
3478\end{array}$ & 2,3 \\
\hline $\begin{array}{l}354 \\
\text { det. }=41888\end{array}$ & $\begin{array}{l}16748 \\
24657 \\
35628\end{array}$ & $\begin{array}{l}2367 \\
1456 \\
1247\end{array}$ & $\begin{array}{l}1237 \\
1345 \\
2345\end{array}$ & $\begin{array}{l}1234 \\
1378 \\
3678\end{array}$ & $\begin{array}{l}1358 \\
1568\end{array}$ & 5,7 \\
\hline $\begin{array}{l}355 \\
\text { det. }=43208\end{array}$ & $\begin{array}{l}35628 \\
16748 \\
13857\end{array}$ & $\begin{array}{l}2467 \\
2367 \\
2456\end{array}$ & $\begin{array}{l}1456 \\
1247 \\
1237\end{array}$ & $\begin{array}{l}1345 \\
2345 \\
1234\end{array}$ & $\begin{array}{l}3678 \\
1568\end{array}$ & 5,7 \\
\hline $\begin{array}{l}356 \\
\text { det. }=51847\end{array}$ & $\begin{array}{l}13658 \\
24657 \\
14728\end{array}$ & $\begin{array}{l}2367 \\
2356 \\
1456\end{array}$ & $\begin{array}{l}1237 \\
1345 \\
2345\end{array}$ & $\begin{array}{l}1234 \\
1378 \\
3678\end{array}$ & $\begin{array}{l}1468 \\
4678\end{array}$ & 3,4 \\
\hline $\begin{array}{l}401 \\
\text { det. }=29760\end{array}$ & $\begin{array}{l}12645 \\
35618 \\
1257\end{array}$ & $\begin{array}{l}1247 \\
1346 \\
1357\end{array}$ & $\begin{array}{l}1347 \\
2346 \\
2368\end{array}$ & $\begin{array}{l}2568 \\
2578 \\
3578\end{array}$ & $\begin{array}{l}2348 \\
2478 \\
3478\end{array}$ & 6,7 \\
\hline $\begin{array}{l}416 \\
\text { det. }=40768\end{array}$ & $\begin{array}{l}16748 \\
35628 \\
2467\end{array}$ & $\begin{array}{l}2367 \\
2456 \\
1456\end{array}$ & $\begin{array}{l}1247 \\
1237 \\
1345\end{array}$ & $\begin{array}{l}2345 \\
1234 \\
1378\end{array}$ & $\begin{array}{l}3678 \\
1358 \\
1568\end{array}$ & 5,7 \\
\hline
\end{tabular}


TABLE 1 (Continued)

\begin{tabular}{|c|c|c|c|c|c|c|c|}
\hline $\begin{array}{l}\text { Case } S_{i} \\
i=\end{array}$ & & & 3-cells & & & & Non contractible at \\
\hline $\begin{array}{l}487 \\
\text { det. }=211023\end{array}$ & $\begin{array}{r}24657 \\
23768 \\
1367\end{array}$ & $\begin{array}{l}1467 \\
2356 \\
1356\end{array}$ & $\begin{array}{l}1456 \\
1247 \\
2345\end{array}$ & $\begin{array}{l}1278 \\
1378 \\
1248\end{array}$ & $\begin{array}{l}2348 \\
1358 \\
1458\end{array}$ & 3458 & 1,2 \\
\hline $\begin{array}{l}488 \\
\text { det. }=214660\end{array}$ & $\begin{array}{r}23768 \\
13857 \\
2467\end{array}$ & $\begin{array}{l}1367 \\
1467 \\
2456\end{array}$ & $\begin{array}{l}2356 \\
1356 \\
1456\end{array}$ & $\begin{array}{l}1247 \\
2345 \\
1278\end{array}$ & $\begin{array}{l}1248 \\
2348 \\
1458\end{array}$ & 3458 & 6,8 \\
\hline $\begin{array}{l}489 \\
\text { det. }=241808\end{array}$ & $\begin{array}{r}34718 \\
23658 \\
1234\end{array}$ & $\begin{array}{l}1237 \\
1267 \\
1256\end{array}$ & $\begin{array}{l}1245 \\
1457 \\
1567\end{array}$ & $\begin{array}{l}2345 \\
3456 \\
4567\end{array}$ & $\begin{array}{l}2378 \\
2678 \\
3468\end{array}$ & 4678 & 2,4 \\
\hline $\begin{array}{l}490 \\
\text { det. }=262300\end{array}$ & $\begin{array}{r}16728 \\
45638 \\
1234\end{array}$ & $\begin{array}{l}1237 \\
1256 \\
1245\end{array}$ & $\begin{array}{l}1347 \\
1457 \\
2345\end{array}$ & $\begin{array}{l}2356 \\
2367 \\
3467\end{array}$ & $\begin{array}{l}1568 \\
1578 \\
4578\end{array}$ & 4678 & 5,7 \\
\hline $\begin{array}{l}506 \\
\text { det. }=236288\end{array}$ & $\begin{array}{r}13857 \\
2467 \\
2367\end{array}$ & $\begin{array}{l}1367 \\
1467 \\
2456\end{array}$ & $\begin{array}{l}2356 \\
1356 \\
1456\end{array}$ & $\begin{array}{l}1247 \\
2345 \\
1278\end{array}$ & $\begin{array}{l}2378 \\
1248 \\
2348\end{array}$ & $\begin{array}{l}1458 \\
3458\end{array}$ & 6,8 \\
\hline $\begin{array}{l}519 \\
\text { det. }=897117\end{array}$ & $\begin{array}{r}16758 \\
1256 \\
1245\end{array}$ & $\begin{array}{l}1347 \\
1457 \\
2345\end{array}$ & $\begin{array}{l}2356 \\
2367 \\
3467\end{array}$ & $\begin{array}{l}3456 \\
4567 \\
1248\end{array}$ & $\begin{array}{l}1348 \\
2348 \\
1378\end{array}$ & $\begin{array}{l}2378 \\
1268 \\
2678\end{array}$ & 2.7 \\
\hline $\begin{array}{l}520 \\
\text { det. }=924672\end{array}$ & $\begin{array}{r}23678 \\
1234 \\
3456\end{array}$ & $\begin{array}{l}1256 \\
1237 \\
1347\end{array}$ & $\begin{array}{l}3467 \\
4567 \\
2567\end{array}$ & $\begin{array}{l}1257 \\
1457 \\
2348\end{array}$ & $\begin{array}{l}1248 \\
3568 \\
3458\end{array}$ & $\begin{array}{l}1268 \\
1568 \\
1458\end{array}$ & 7,8 \\
\hline
\end{tabular}

det. is the determinant of the edge-valence matrix. Every 3-cell with five vertices is a double-tetrahedron.

6. Classification: $\mathbf{5 9 6}$ polytopal spheres. The following three theorems are particular cases of more general theorems stated and proved in [5], and are used here to show that 596 of our 617 spheres are polytopal.

THeOREM 3. Let $Q \subset R^{4}$ be a 4-polytope, let $L$ be an edge of $Q$ and let $A_{1}, \ldots, A_{m}$ be all the facets of $Q$ in $\operatorname{st}(L, Q)$ in their natural cyclic order, that is, $A_{i} \cap A_{i+1}, 1 \leq i \leq m$ (where $A_{m+1}=A_{1}$ ) is the 2-face common to $A_{i}$, $A_{i+1}$ and contains $L$. Then for every $n \leq m$ there are points $x_{0}, x_{1}, x_{2}$ in $R^{4}$ such that, with respect to $Q$ :

$x_{0}$ lies beyond $A_{1}, \ldots, A_{n}$ and beneath all the other facets of $Q$;

$x_{1}$ lies in aff $A_{1}$ beyond $A_{2}, \ldots, A_{n}$ and beneath all the other facets of $Q$ ( provided $n \geq 2$ );

$x_{2}$ lies in aff $A_{1} \cap$ aff $A_{n}$, beyond $A_{2}, \ldots, A_{n-1}$ and beneath all the other facets of $Q$ ( provided $n \geq 3$ );

and, for each $0 \leq i \leq 2$, vert $\operatorname{conv}\left(Q \cup\left\{\dot{x}_{i}\right\}\right)=\operatorname{vert} Q \cup\left\{x_{i}\right\}$. 
RemarK. Note that if in Theorem 3, case $x_{0}$, we take $n=1$, we get the frequently used (and trivial) assertion that for very facet $F$ of a 4-polytope $Q$ there is a point which lies beyond $F$ and beneath all the other facets of $Q$.

THEOREM 4. With the conventions of Theorem 3, let $n \leq m$, let $y$ be a vertex of $L$ and let $\mathscr{Q}$ be the set of all the facets of $Q$ other than $A_{1}, \ldots, A_{n}$ which contain $y$. Then there are points $x_{0}^{\prime}, x_{1}^{\prime}, x_{2}^{\prime}$ in $R^{4}$ such that, with respect to $Q$ :

$x_{0}^{\prime}$ lies beyond every $F \in \mathcal{Q}$ and beneath all the other facets of $Q$;

$x_{1}^{\prime}$ lies in aff $A_{1}$, beyond every $F \in \mathscr{Q}$ and beneath all the other facets of $Q$;

$x_{2}^{\prime}$ lies in aff $A_{1} \cap$ aff $A_{n}$, beyond every $F \in \mathscr{Q}$ and beneath all the other facets of $Q$ ( provided $n \geq 2$ );

and, for each $0 \leq i \leq 2, \operatorname{vert} \operatorname{conv}\left(Q \cup\left\{x_{i}^{\prime}\right\}\right)=\operatorname{vert} Q \cup\left\{x_{i}^{\prime}\right\}$.

TheOREM 5. Let $Q \subset R^{4}$ be a 4-polytope, and let $A_{0}$ be a facet of $Q$ which contains a vertex $p$ whose valence in $A_{0}$ is 3 and whose valence in $Q$ is $\geq 5$. Let $A_{1}, A_{2}, A_{3}$ be the facets of $Q$ adjacent to $A_{0}$ (that is, sharing $a$ common 2-face with $A_{0}$ ) and containing $p$, and let $D=\{1,2,3\}$. Then for every two disjoint subsets $S, T$ of $D$ (including the cases $S=\varnothing, T=\varnothing$ ) there are points $x_{1}, x_{2} \in R^{4}$ such that, with respect to $Q$ :

$x_{1}$ lies in $\bigcap_{t \in T}$ aff $A_{t}$, beyond $A_{0}$, beyond every $A_{s}, s \in S$, and beneath all the other facets of $Q$;

$x_{2}$ lies in $\bigcap_{t \in T}$ aff $A_{t}$, beyond all the facets of $Q$ other than $A_{0}, A_{1}, A_{2}$, $A_{3}$, which contain $p$, beyond $A_{r}(r \in D \backslash(S \cup T))$, and beneath all the other facets of $Q$;

and for $i=1,2, \operatorname{vert}\left(\operatorname{conv}\left(Q \cup\left\{x_{i}\right\}\right)\right)=\operatorname{vert} Q \cup\left\{x_{i}\right\}$

REMARK. In Theorem 5, the condition that the valence of $p$ in $Q$ is $\geq 5$ is superfluous in case $S \cup T \neq D$.

As already mentioned, for each $S_{i}, 1 \leq i \leq 520$, and for each vertex $x$ of $S_{i}$, we computed all the simplicial refills for $S_{i}$ at $x$. As an example, consider the sphere $S_{195}$ (see Table 2). At its vertex 2, it has a refill $R_{1}$, the 3-cells of which are 1567, 3568, 4678, 5678, -1467, -3468 (the negative sign before a 3-cell denotes that that 3-cell is in $\left.C=C\left(R_{1}\right)\right)$. All those 3 -cells contain the vertex 6 . The 3 -cells in ast $\left(2, S_{195}\right)$ which contain the vertex 6 are 1346 and 1356. We now use the second part of Theorem 5 to show that $S_{195}$ is polytopal. 
TABLE 2

Some polytopal quasisimplicial 3-spheres with eight vertices

\begin{tabular}{lrrrrl}
\hline $\begin{array}{l}\text { Case } S_{i} \\
i=\end{array}$ & \multicolumn{5}{c}{ 3-cells } \\
\hline 15 & 12768 & 12546 & 2345 & 1378 & \\
det. $=112$ & 13657 & 23847 & 2356 & & \\
127 & 13458 & 2367 & 1248 & & \\
& 23748 & 26857 & 1345 & 3467 & \\
det. $=1426$ & 35618 & 1245 & 1346 & 2358 & \\
141 & 24617 & 1256 & 2345 & 3678 & \\
& 12378 & 1345 & 1367 & 2356 & 2348 \\
det. $=0$ & 1256 & 1356 & 2367 & 1248 & \\
195 & 1245 & 1267 & 2345 & 1348 & \\
& 34718 & 1256 & 1356 & 2358 & 2478 \\
det. $=500$ & 12467 & 1257 & 1357 & 2578 & \\
325 & 23468 & 1346 & 2356 & 3578 & \\
& 13658 & 2367 & 1456 & 1234 & 1238 \\
det. $=14736$ & 24657 & 1467 & 1345 & 1678 & 2378 \\
617 & 12748 & 2356 & 2345 & 3678 & \\
& 578631 & 1467 & 1456 & 1278 & 2348 \\
det. $=53760$ & 2467 & 2456 & 1247 & 2378 & 1458 \\
\hline
\end{tabular}

det. is the determinant of the edge-valence matrix. Every 3-cell with five vertices is a double-tetrahedron. The 3-cell with six vertices (in Case 617) is an octahedron.

The contraction $S_{195}^{2}=\operatorname{ast}\left(2, S_{195}\right) \cup R_{1}$ is a 3 -sphere with seven vertices, and therefore it is polytopal. Let $Q$ be any 4-polytope which realizes $S_{195}^{2}$, with the same labelling of the vertices. To conform to the notation of Theorem 5, let $A_{0}=1346, A_{1}=1467, A_{2}=3468, A_{3}=1356$, $\mathscr{D}=\{1567,3568,4678,5678\}, T=\{1,2\}, S=\{3\}$ and $p=6$.

The second part of Theorem 5 and Grünbaum's theorem now guarantee the existence of a point $x_{2}$ in $R^{4}$ and the existence of a combinatorial equivalence $\varphi$, such that the polytope $P=\operatorname{conv}\left(Q \cup\left\{x_{2}\right\}\right)$ has eight vertices $1, x_{2}, 3,4, \ldots, 8$ and $\varphi$ is a combinatorial equivalence between $S_{195}$ and bd $P$ such that $\varphi(i)=i$ for every $1 \leq i \leq 8, i \neq 2$, and $\varphi(2)=x_{2}$. Therefore $S_{195}$ is polytopal.

$S_{195}$ has another simplicial refill $R_{2}$ at the vertex 2, the 3-cells of which are $1567,3568,4568,4578,4567,-1467,-3468$. But this refill is not useful for our purpose, since there is no vertex common to all the 3-cells in $R_{2}$, and therefore none of the Theorems 3, 4, 5 can be used here. One would like to correct the situation by gluing together the 3-cells 4578 and 4567 , that is, by replacing in $R_{2}$ these two 3-cells by the type A cell 45768 , 
thus obtaining a refill all of whose 3-cells contain the vertex 6 . But this will not yield a refill, since the edge 68 would appear here both as an edge in 3468 and as a missing (interior) edge in the new 3-cell 45768.

On the other hand, $S_{195}$ has two simplicial refills at vertex 8 , the 3-cells of one of which are 2347, 2357, -1347, -2346. Here 2347 and 2357 can be glued together to yield the (type A) cell 23745, and now the third part of Theorem 3 can be evoked, with $L=34, n=3, A_{1}=1347$, $A_{2}=23745, A_{3}=2346$, to yield once again that $S_{195}$ is polytopal.

In a similar manner we proved that 499 of the 520 spheres $S_{i}$, $1 \leq i \leq 520$, are polytopal. In each of those cases there was a simplicial refill which, together with one of the Theorems 3, 4, 5, did the job. Since we have already seen that 18 of the spheres $S_{i}, 1 \leq i \leq 520$, are not polytopal. There remain just three undecided spheres in this group and we deal with them in the next section. They are $S_{15}, S_{127}$ and $S_{325}$.

As for the remaining 97 spheres $S_{i}, 521 \leq i \leq 617$, each of which has a 3-cell of either type $\mathrm{B}$ or $\mathrm{C}$, the process of finding all the refills at all the vertices is slightly more complicated-because of the non-uniqueness of the admissible 3-cell arising from a cell of type B or C-and we did not attempt it. However, for each of these spheres we found a vertex and a refill at that vertex, for which one of the Theorems 3, 4, 5 could be used to prove that the sphere is indeed polytopal. We now describe one such example.

Consider the last sphere $S_{617}$ (see Table 2). We will find a refill at the vertex 1 . Besides tetrahedra, st $\left(1, S_{617}\right)$ contains the octahedron 578631 (the notation is canonic, as in Figure 1, type C). This octahedron admits three admissible 3-cells at its vertex 1: a type B cell 35768, a type B cell 36857 , and a pyramid 56783 with apex 3 . Now, each of these admissible

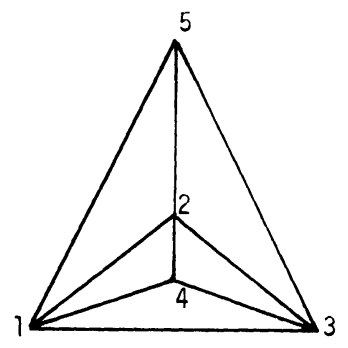

Type A: 12345

double tetrahedron

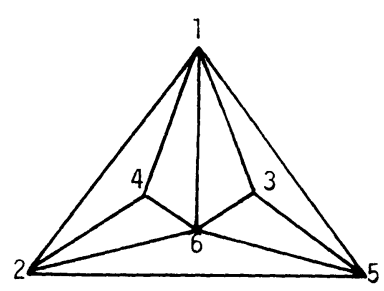

Type B: 123456

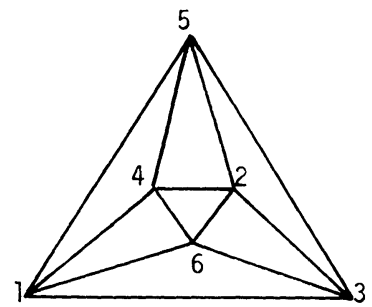

Type C: 123456

Octahedron 
3-cells can be completed into a refill from which Theorems 3, 4 and 5 establish the polytopality of $S_{617}$. For the refill constructed from the type B cell $A_{1}=35768$, the remaining 3-cells are $A_{0}=4578, A_{2}=2478, A_{3}=$ 4567, which conforms to the first part of Theorem 5 with $p=7, T=\{1\}$, $S=\{2,3\}$. For the refill constructed from the type B cell $A_{1}=36857$, the remaining 3-cells are $A_{0}=4678, A_{2}=2478, A_{3}=4568$, and this too conforms to the first part of Theorem 5, with $p=8, T=\{1\}, S=\{2,3\}$. Finally, for the refill constructed from the pyramid $A_{1}=56783$ (with apex 3 ), the other 3-cells are a pyramid $A_{3}=56784$ with apex 4 and a tetrahedron $A_{2}=2478$. This conforms to the second part of Theorem 3 with $L=78, m=4, n=3$.

7. Classification: The last three cases. We now turn our attention to the three remaining spheres $S_{15}, S_{127}, S_{325}$ (see Table 2) which are undecided, and we show that they are polytopal.

The sphere $S_{15}$. To see that none of the Theorems 3, 4, 5 can be used here, we note that for every vertex $x$ other than 5,6 , and for every refill $R$ of $S_{15}$ at $x$, the 3-cells in $\mathcal{C}=\mathcal{C}(R)$ (that is, the 3-cells in $R$ whose existence is required by part (iv) of Definition 1) do not share a common vertex. At vertex 5, the only refill is composed of the 3-cells 1346, 2346, $-1367,-1348,-1246$, but these five tetrahedra do not share a common vertex, and 1346 cannot be glued to 2346 to change the situation. A similar situation occurs at vertex 6 , where the only refill consists of the 3-cells 1257, 2357, -1278, -1357, -1245.

With the refill for $S_{15}$ at 5 mentioned above, let $Q$ be any 4-polytope realizing $S_{15}^{5}$, the contraction of $S_{15}$ at 5 , and containing the origin in its interior. Recall that every 5-vertex facet in $S_{15}$, and therefore also in $Q$, is a double tetrahedron. The facets of $Q$ are as follows:

$$
\begin{array}{lllll}
A_{1}=1346 & C_{1}=1246 & C_{3}=1367 & B_{2}=1248 & B_{4}=23847 \\
A_{2}=2346 & C_{2}=1348 & B_{1}=1378 & B_{3}=2367 & B_{5}=12768
\end{array}
$$

Our goal is to prove the existence of a point $x$ in $R^{4}$ which, with respect to $Q$, lies in $\cap_{k=1}^{3}$ aff $C_{k}$, beyond each $A_{i}, i=1,2$ and beneath each $B_{j}, 1 \leq j \leq 5$. Let $Q^{*}$ be the 4-polytope polar to $Q$, and for every $i$-face ( $0 \leq i \leq 3$ ) $F$ of $Q$ denote by $F^{*}$ the corresponding (3-i)-face of $Q^{*}$. Our goal will be achieved by finding a hyperplane $H$ in $R^{4}$ which contains the vertices $C_{k}^{*}, 1 \leq k \leq 3$, of $Q^{*}$ and strictly separates the vertices $A_{i}^{*}$, $i=1,2$ from the vertices $B_{j}^{*}, 1 \leq j \leq 5$, since $x$ can be taken as the point polar to $H$. 

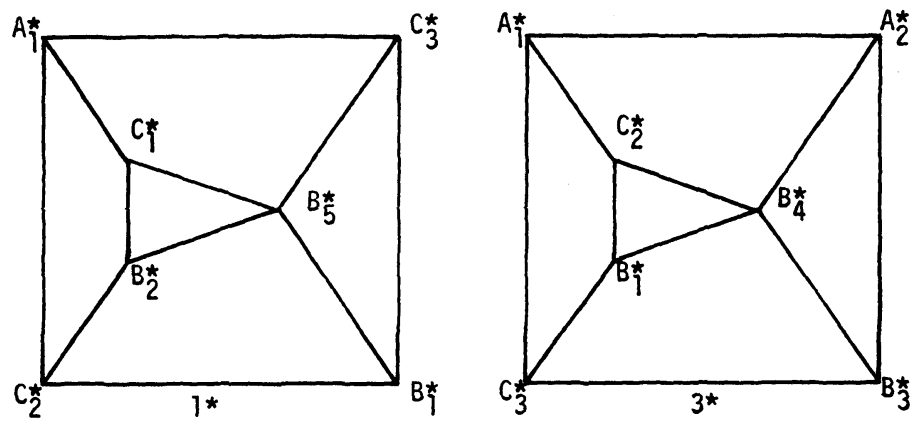

The facets $1^{*}, 3^{*}$ of $Q^{*} \approx\left(S_{15}^{5}\right)^{*}$

FIGURE 2

Figure 2 depicts Schlegel diagrams of the facets $1^{*}$ and $3^{*}$ of $Q^{*}$. The hyperplane aff $1^{*}$ supports $Q^{*}$ and therefore does not contain the vertex $A_{2}^{*}$. Let $y$ be any point interior to the edge $A_{2}^{*} B_{3}^{*}$, which is not in $F=\operatorname{aff}\left\{C_{1}^{*}, C_{2}^{*}, C_{3}^{*}\right\}$. We claim that $H=\operatorname{aff}\left\{y, C_{1}^{*}, C_{2}^{*}, C_{3}^{*}\right\}$ is the desired hyperplane.

Indeed, let $H^{+}, H^{-}$be the open halfspaces of $R^{4}$ determined by $H$ so that $A_{2}^{*} \in H^{-}$, and let $G=H \cap$ aff $3^{*}$. A study of the 2-face $A_{1}^{*} A_{2}^{*} B_{3}^{*} C_{3}^{*}$ reveals that, in aff $3^{*}, G$ strictly separates $A_{1}^{*}$ and $A_{2}^{*}$ from $B_{3}^{*}$. A study of the 2-face $A_{1}^{*} C_{2}^{*} B_{1}^{*} C_{3}^{*}$ reveals that, in aff $3^{*}, G$ strictly separates $A_{1}^{*}$ from $B_{1}^{*}$, and a study of the 2 -face $B_{1}^{*} B_{4}^{*} B_{3}^{*} C_{3}^{*}$ reveals that, in aff $3^{*}, B_{1}^{*}, B_{3}^{*}$, and $B_{4}^{*}$ lie on the same side of $G$. Thus $A_{1}^{*}, A_{2}^{*} \in H^{-}$and $B_{1}^{*}, B_{3}^{*}, B_{4}^{*} \in$ $H^{+}$. Now consider the facet $1^{*}$ of $Q^{*}$. Since $F=H \cap$ aff $1^{*}$ and, in aff $1^{*}, F$ strictly separates $A_{1}^{*}$ from $B_{1}^{*}, B_{2}^{*}$ and $B_{5}^{*}$, it follows that $B_{2}^{*}, B_{5}^{*} \in H^{+}$.

We thus conclude that $S_{15}$ is polytopal.

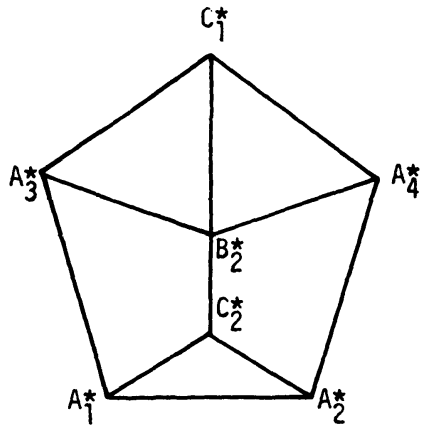

The facet $8^{*}$ of $Q^{*} \approx\left(S_{127}^{3}\right)^{*}$

FIGURE 3 
The sphere $S_{127}$. A refill for $S_{127}$ at vertex 3 consists of the 3-cells $A_{1}=1458, A_{2}=1468, A_{3}=2458, A_{4}=4678, C_{1}=2478, C_{2}=1568$. In addition, $S_{127}^{3}$ contains the 3-cells $B_{1}=24617, B_{2}=26857, B_{3}=1245$, $B_{4}=1256$. Let $Q$ be any 4-polytope realizing $S_{127}^{3}$ with the origin of $R^{4}$ in its interior, and let $Q^{*}$ be the polytope polar to $Q$. Figure 3 depicts a Schlegel diagram of the facet $8^{*}$ of $Q^{*}$.

As in the case of $S_{15}$, it is sufficient to find a hyperplane $H$ which contains $C_{1}^{*}, C_{2}^{*}$ and strictly separates $A_{1}^{*}, A_{2}^{*}, A_{3}^{*}, A_{4}^{*}$ from $B_{1}^{*}, B_{2}^{*}, B_{3}^{*}$, $B_{4}^{*}$. Let $F=\operatorname{aff}\left\{C_{1}^{*}, C_{2}^{*}, A_{4}^{*}\right\}$ and let $F^{+}, F^{-}$be the open 3-dimensional half-spaces determined by $F$ in aff $8^{*}$ so that $A_{3}^{*} \in F^{-}$. Then clearly $A_{1}^{*}, A_{2}^{*} \in F^{-}$. As $F$ separates $A_{2}^{*}$ from $B_{2}^{*}, B_{2}^{*} \in F^{+}$. Thus a slight rotation in aff $8^{*}$ of $F$ about the line aff $\left\{C_{1}^{*}, C_{2}^{*}\right\}$ yields a plane $G$ which, in aff $8^{*}$, contains $C_{1}^{*}, C_{2}^{*}$ and strictly separates $B_{2}^{*}$ from $A_{1}^{*}, A_{2}^{*}, A_{3}^{*}$ and $A_{4}^{*}$. Finally, a slight rotation of aff $8^{*}$ about $G$ yields the desired hyperplane $H . S_{127}$ is therefore polytopal.

The sphere $S_{325}$. A refill for $S_{325}$ at vertex 8 consists of the 3-cells $A_{1}=1237, A_{2}=1367, C_{1}=1356, C_{2}=1247$. In addition, $S_{325}^{8}$ contains the 3-cells $B_{1}=24657, B_{2}=2367, B_{3}=1467, B_{4}=2356, B_{5}=1456$, $B_{6}=1345, B_{7}=2345, B_{8}=1234$. Let $Q$ be any 4-polytope realizing $S_{325}^{8}$ with the origin of $R^{4}$ in its interior, and let $Q^{*}$ be the polytope polar to $Q$. Figure 4 depcits a Schlegel diagram of the facet $1^{*}$ of $Q^{*}$.

To prove the polytopality of $S_{325}$ it is sufficient here, as before, to find a hyperplane $H$ which contains $C_{1}^{*}, C_{2}^{*}$ and strictly separates $A_{1}^{*}, A_{2}^{*}$ from $B_{J}^{*}, 1 \leq j \leq 8$. Let $F=\operatorname{aff}\left\{C_{1}^{*}, C_{2}^{*}, B_{3}^{*}\right\}$ and let $F^{+}, F^{-}$be the two open 3-dimensional half-spaces determined by $F$ in aff $1^{*}$, so that $A_{2}^{*} \in F^{-}$. Then clearly $B_{5}^{*} \in F^{+}$. But, in aff $1^{*}, B_{5}^{*}, B_{6}^{*}, B_{8}^{*}$ are on the same side of $F$, hence $B_{6}^{*}, B_{8}^{*} \in F^{+}$. Since $A_{2}^{*}, A_{1}^{*}$ are on the same side of $F, A_{1}^{*} \in F^{-}$. Thus, a slight rotation in aff $1^{*}$ of $F$ about the line aff $\left\{C_{1}^{*}, C_{2}^{*}\right\}$ yields a

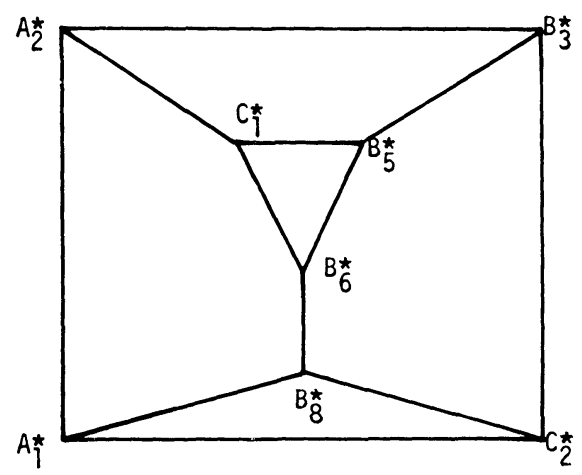

The facet $1^{*}$ of $Q^{*} \approx\left(S_{325}^{8}\right)^{*}$

FigURE 4 
plane $G$ which, in aff $1^{*}$, contains $C_{1}^{*}, C_{2}^{*}$ and strictly separates $A_{1}^{*}, A_{2}^{*}$ from $B_{3}^{*}, B_{5}^{*}, B_{6}^{*}$ and $B_{8}^{*}$. Finally, a slight rotation of aff $1^{*}$ about $G$ yields the desired hyperplane $H$. Thus, $S_{325}$ too is polytopal.

This ends the proof of the main result in the present work, namely:

THEOREM 6. There are precisely 661 quasisimplicial 3-spheres with eight vertices, and precisely 20 of them are not polytopal.

\section{REMARKS.}

1. The sphere $S_{617}$, discussed in detail in $\$ 6$ and whose polytopality follows from our general arguments, is actually the 3-sphere discussed by Kleinschmidt in [18], and shown by him to be a minimal polytope having a facet of non-arbitrary shape. Kleinschmidt proves the polytopality of this sphere by using a theorem by Shephard.

2. As already mentioned, 10 of our 18 non-polytopal spheres appear in [20]. They are the following, where the number in parentheses denotes the notation in [20]: 125(15), 194(6), 348(16), 349(24), 401(7), 487(25), 488(17), 506(8), 519(26) and 520(18). Using our terminology and Schulz's labelling of the vertices, the proof for the non-polytopality of those spheres (and also of Barnette's and Grünbaum's spheres, in Schulz's labelling) is common and brief. If $S$ is any of those spheres, then the 2-faces in bd ast $(8, S)$ are the triangles 123, 125, 134, 145, 236, 256, 456. Since 16, 24 and 35 are either edges or missing edges of valence 1 in ast $(8, S)$, and 7 is an interior vertex there, none of them should appear in a refill for $S$ at 8 . But then even the first triangle, 123, cannot be completed into a 3-simplex. Thus all those spheres are not contractible at the vertex 8 , and therefore are not polytopal.

3. It is a fact for which we have no explanation, that each of the 20 non-polytopal quasisimplicial 3-spheres with eight vertices is not contractible at precisely two vertices. For our 18 non-simplicial cases these two vertices are given in Table 1. Barnette's sphere is not contractible at the vertices 7, 8 (in the original labelling of [10]), and Grünbaum's sphere is not contractible at the vertices 4, 6 (in the original labelling of [15]). Hopefully, in the second part of this work we will find if for every nonpolytopal 3-sphere $S$ with eight vertices, $S$ is not contractible at some vertex, and if so, that it is not contractible at precisely two vertices. There is however, a non-polytopal simplicial 3-sphere with nine vertices that is contractible at each vertex (the non-polytopal sphere in [9]), as well as many such 3-spheres with 10 vertices ([3]). On the other hand, there is a 3-sphere with ten vertices which is not contractible at all, that is, there is no vertex at which this sphere is contractible (see [2]). 
4. Each of our 18 non-polytopal spheres has the same number of simplicial refills ( 1 or 2 ) at each of the six vertices at which it is contractible. This probably reflects some symmetry properties of those spheres. As for the entire list of 520 3-spheres whose 3-cells are just tetrahedra and double-tetrahedra-which are exactly the spheres for which we computed all the simplicial refills at all the vertices-the number of simplicial refills usually varies from one vertex to the other in the same sphere. Many spheres admit just one simplicial refill at each vertex, but there are also spheres that admit many simplicial refills at the same vertex. There are three spheres $\left(S_{61}, S_{63}, S_{249}\right)$ that admit 16 simplicial refills at some of their vertices, two spheres $\left(S_{144}, S_{145}\right)$ that admit 23 simplicial refills at some of their vertices, and there is a sphere ( $S_{141}$, see Table 2 ) which admits 35 simplicial refills at vertices 1,2 , and 3 .

5. In $\$ 6$ we used Theorems 3,4 and 5 to prove the polytopality of most of our spheres. Those theorems, however, are not totally "disjoint". The case $S \cup T \neq D$ in Theorem 5 is "covered" also by Theorems 3, 4.

6. The non-polytopal 3-spheres discussed in the present work tend, with exceptions, to have high determinants, often the highest determinants (that is, the determinants of the edge-valence matrices) among the 3-spheres which share the same number of vertices and the same number of 3-cells, particularly when the number of 3-cells is even. The same phenomenon has been observed in the simplicial 3-spheres with nine vertices ([8]) and in the neighborly 3-spheres with 10 vertices ([3]) - that is, among all the 3 -spheres of which we have complete catalogues.

\section{REFERENCES}

[1] A. Altshuler, Combinatorial 3-manifolds with few vertices, J. Combinatorial Theory (A), 16 (1974), 165-173.

[2] A peculiar triangulation of the 3-sphere, Proc. Amer. Math. Soc., 54 (1976), 449-452.

[3] N Neighborly 4-polytopes and neighborly combinatorial 3-manifolds with 10 vertices, Canad. J. Math., 29 (1977), 400-420.

[4] , 3-Pseudominfolds with preassigned links, Trans. Amer. Math. Soc., 241 (1978), 213-237.

[5] A. Altshuler and I. Shemer, Construction theorems for convex polytopes, to appear.

[6] A. Altshuler and L. Steinberg, Neighborly 4-polytopes with nine vertices, J. Combinatorial Theory (A), 15 (1973), 270-287.

[7] N Neighborly combinatorial 3-manifolds with nine vertices, Discrete Math., 8 (1974), 113-137.

[8] An enumeration of combinatorial 3-manifolds with nine vertices, Discrete Math., 16 (1976), 91-108.

[9] A. Altshuler, L. Steinberg and J. Bokowski, The classification of simplicial 3-spheres with nine vertices into polytopes and nonpolytopes, Discrete Math., 31 (1980), 115-124. 
[10] D. Barnette, The triangulations of the 3-sphere with up to 8 vertices, J. Combinatorial Theory (A), 14 (1973), 37-53.

[11] U. Betke, Ch. Schulz and J. M. Wills, Bänder und Mäbiusbänder in konvexen Polytopen, Abh. Math. Sem. Hamburg, 47 (1978), 113-127.

[12] G. Danaraj and V. Klee, Shellings of spheres and polytopes, Duke Math. J., 41 (1974), 443-451.

[13] P. J. Federico, Enumeration of polyhedra: The number of 9-hedra. J. Combinatorial Theory, 7 (1969), 155-161.

[14] B. Grünbaum, Convex Polytopes, Interscience, John Wilet and sons, 1967.

[15] B. Grünbaum and V. P. Sreedharan, An enumeration of simplicial 4-polytopes with 8 vertices, J. Combinatorial Theory, 2 (1967), 437-465.

[16] P. Kleinschmidt, Sphären mit wenigen Ecken, Geom. Dedicata, 5 (1976), 307-320.

[17] _ـ Nicht-dualisierbar sphären, Geom. Dedicata, 6 (1977), 511-515.

[18] _ On facets with non-arbitrary shapes, Pacific J. Math., 65 (1976), 97-101.

[19] M. Reif, 4-polytopes with 8 vertices, M. Sc. thesis under the supervision of A. Altshuler, Ben-Gurion University of the Negev, 1979.

[20] Ch. Schulz, Nicht-Polytopale 3-sphären mit 8 ecken, Geom. Dedicata, 13 (1982), 325-329.

[21] __ Geometrische Realisierungen Zellzerlegter Mannigfaltigkeiten in Euklidischen Räumen, Habilitationsschrift, (mimeographed) 1980.

Received July 16, 1982.

Ben GuRion UNIVERSITY OF THE NegeV

BEER SHEVA 84105, ISRAEL 


\section{PACIFIC JOURNAL OF MATHEMATICS \\ EDITORS}

Donald BaBBITT (Managing Editor)

University of California

Los Angeles, CA 90024

Hugo RossI

University of Utah

Salt Lake City, UT 84112

C. C. Moore and Arthur Ogus

University of California

Berkeley, CA 94720
J. DugundiI

Department of Mathematics

University of Southern California

Los Angeles, CA 90089-1113

R. FINN and H. SAMELSON

Stanford University

Stanford, CA 94305

\section{ASSOCIATE EDITORS}
R. ARENS
E. F. BECKENBACH
B. H. NeUMANN
F. WOLF
K. YoshidA (1906-1982)

\section{SUPPORTING INSTITUTIONS}

\author{
UNIVERSITY OF ARIZONA \\ UNIVERSITY OF BRITISH COLUMBIA \\ CALIFORNIA INSTITUTE OF TECHNOLOGY \\ UNIVERSITY OF CALIFORNIA \\ MONTANA STATE UNIVERSITY \\ UNIVERSITY OF NEVADA, RENO \\ NEW MEXICO STATE UNIVERSITY \\ OREGON STATE UNIVERSITY
}

\author{
UNIVERSITY OF OREGON \\ UNIVERSITY OF SOUTHERN CALIFORNIA \\ STANFORD UNIVERSITY \\ UNIVERSITY OF HAWAII \\ UNIVERSITY OF TOKYO \\ UNIVERSITY OF UTAH \\ WASHINGTON STATE UNIVERSITY \\ UNIVERSITY OF WASHINGTON
}

The Supporting Institutions listed above contribute to the cost of publication of this Journal, but they are not owners or publishers and have no responsibility for its content or policies.

Mathematical papers intended for publication in the Pacific Journal of Mathematics should be in typed form or offset-reproduced (not dittoed), double spaced with large margins. Please do not use built up fractions in the text of the manuscript. However, you may use them in the displayed equations. Underline Greek letters in red, German in green, and script in blue. The first paragraph must be capable of being used separately as a synopsis of the entire paper. In particular it should contain no bibliographic references. Please propose a heading for the odd numbered pages of less than 35 characters. Manuscripts, in triplicate, may be sent to any one of the editors. Please classify according to the scheme of Math. Reviews, Index to Vol. 39. Supply name and address of author to whom proofs should be sent. All other communications should be addressed to the managing editor, or Elaine Barth, University of California, Los Angeles, California 90024.

There are page-charges associated with articles appearing in the Pacific Journal of Mathematics. These charges are expected to be paid by the author's University, Government Agency or Company. If the author or authors do not have access to such Institutional support these charges are waived. Single authors will receive 50 free reprints; joint authors will receive a total of 100 free reprints. Additional copies may be obtained at cost in multiples of 50 .

The Pacific Journal of Mathematics is issued monthly as of January 1966. Regular subscription rate: $\$ 132.00$ a year (6 Vol., 12 issues). Special rate: $\$ 66.00$ a year to individual members of supporting institutions.

Subscriptions, orders for numbers issued in the last three calendar years, and changes of address should be sent to Pacific Journal of Mathematics, P.O. Box 969, Carmel Valley, CA 93924, U.S.A. Old back numbers obtainable from Kraus Periodicals Co., Route 100, Millwood, NY 10546.

The Pacific Journal of Mathematics ISSN 0030-8730 is published monthly by the Pacific Journal of Mathematics at P.O. Box 969, Carmel Valley, CA 93924. Application to mail at Second-class postage rates is pending at Carmel Valley, California, and additional mailing offices. Postmaster: Send address changes to Pacific Journal of Mathematics, P. O. Box 969, Carmel Valley, CA 93924.

PUBLISHED BY PACIFIC JOURNAL OF MATHEMATICS, A NON-PROFIT CORPORATION

Copyright $\odot 1984$ by Pacific Journal of Mathematics 


\section{Pacific Journal of Mathematics}

\section{Vol. 113, No. $2 \quad$ April, 1984}

Alan Adolphson, On the Dwork trace formula ...................257

Amos Altshuler and Leon Steinberg, Enumeration of the quasisimplicial

3 -spheres and 4-polytopes with eight vertices .................. 269

Kenneth R. Goodearl, Cancellation of low-rank vector bundles .......... 289

Gary Fred Gruenhage, Ernest A. Michael and Yoshio Tanaka, Spaces

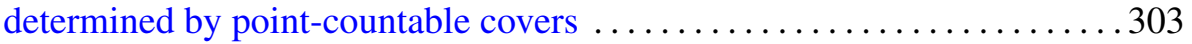

Charles Lemuel Hagopian, Atriodic homogeneous continua .......... 333

David Harbater, Ordinary and supersingular covers in characteristic $p$. . . 349

Domingo Antonio Herrero, Continuity of spectral functions and the lakes

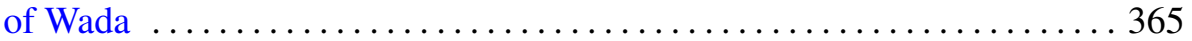

Donald William Kahn, Differentiable approximations to homotopy resolutions and framed cobordism ....................... 373

K. McGovern, On the lifting theory of finite groups of Lie type $\ldots \ldots \ldots . \ldots 383$

C. David (Carl) Minda, The modulus of a doubly connected region and the geodesic curvature-area method ............................. 395

Takuo Miwa, Complexes are spaces with a $\sigma$-almost locally finite base $\ldots . .407$

Ho Kuen Ng, Finitely presented dimension of commutative rings and modules

Roger David Nussbaum, A folk theorem in the spectral theory of

$C_{0}$-semigroups

J. S. Okon, Prime divisors, analytic spread and filtrations

Harold Raymond Parks, Regularity of solutions to elliptic isoperimetric problems

R. Sitaramachandra Rao and M. V. Subba Rao, Transformation formulae for multiple series

Daniel Ruberman, Imbedding punctured lens spaces and connected sums

Uri Srebro, Deficiencies of immersions 